\title{
Specular Reflectance Measurements of Dielectric Plates in Millimeter Frequency Range
}

\author{
Jin-Seob Kang ${ }^{1, *} \cdot$ Jeong-Hwan Kim ${ }^{1} \cdot$ Kwang Yong Kang ${ }^{2} \cdot$ Dae Hwan Yoon ${ }^{3} \cdot$ Sung Won Park
}

\begin{abstract}
This paper describes specular reflectance measurements of dielectric plates in three waveguide frequency bands: D-band (110-170 GHz), G-band (140-220 GHz), and J-band (220-325 GHz). The transmit (Tx) part of the proposed specular reflectance measurement system is stationary, while the receive $(\mathrm{Rx})$ part and the material under test (MUT) holder are concentric-rotating with a 2:1 speed ratio for specular reflectance measurements. In specular reflectance measurements, the first step measures the specular reflection coefficients of an MUT and a metal plate on the MUT holder located at the center of the Tx and Rx parts, and the second step calculates the specular reflectance defined by the specular reflection power (i.e., intensity) of the MUT normalized to that of the metal plate. Multiple reflection effects between the Tx and Rx antennas and the MUT on the measured specular reflectance are minimized by averaging out the multiple specular reflectances measured with changing the separation distance between the two antennas by $\lambda / 8$ intervals. Measurement results of the perpendicular-polarized specular reflectance of commonly used dielectric plates are verified by comparing those with the analytic results and show that the results measured over the overlapped frequency range of the D-/G-bands and at the boundary frequency of the GJ-bands agree well with the results for the other band, respectively.
\end{abstract}

Key Words: Dielectric Plate, Material Measurements, Millimeter-Wave, Multiple Reflections, Specular Reflectance.

\section{INTRODUCTION}

Recently, uses and applications of electromagnetic (EM) materials, devices, and systems have been becoming more popular in the fields of both the natural sciences, such as physics, chemistry, biology, earth science, and astronomy, and the application sciences, such as telecommunications, military, defense, security, transportation, and medicine. Their bandwidths have been broadening and operating frequency bands have been moving to the submillimeter frequency range. Measurements of the electrical properties of EM materials have gained considerable im- portance, particularly in the millimeter and submillimeter frequency ranges, as material parameters are fundamental parameters in the natural and application sciences fields $[1,2]$.

Various methods have been developed in millimeter and submillimeter frequency ranges to measure material properties (usually complex permittivity and permeability), e.g., the open resonator [3, 4], free space [5-10], and reflection ellipsometry methods [11-13]. The open resonator method provides accurate material properties at discrete resonance frequencies. The free space method is based on the quasi-optic approach where the material under test (MUT) is coupled to transmit (Tx) and

Manuscript received July 18, 2017 ; Revised January 10, 2018 ; Accepted January 17, 2018. (ID No. 20170718-031J)

${ }^{1}$ Center for Electromagnetic Metrology, Korea Research Institute of Standards and Science (KRISS), Daejeon, Korea.

${ }^{2}$ Panoptics, Seongnam, Korea.

${ }^{3}$ National Radio Research Agency (RRA), Naju, Korea.

"Corresponding Author: Jin-Seob Kang (e-mail: jinskang@kriss.re.kr)

This is an Open-Access article distributed under the terms of the Creative Commons Attribution Non-Commercial License (http://creativecommons.org/licenses/by-nc/4.0) which permits unrestricted non-commercial use, distribution, and reproduction in any medium, provided the original work is properly cited.

(c) Copyright The Korean Institute of Electromagnetic Engineering and Science. All Rights Reserved. 
receive $(\mathrm{Rx})$ antennas that are connected to the test ports of an $S$-parameter measuring instrument through two focused Gaussian beams generated by spot-focused horn lens antennas $[5,6]$, corrugated horn antennas with mirrors [7-9], and planar focusing arrays [10]. The reflection ellipsometry method is a wellestablished method for material characterization in optics and has been recently adapted to millimeter frequencies to determine material properties from the change of the polarization state of the wave reflected by an MUT that is illuminated by the linear-polarized wave radiated by a $\mathrm{Tx}$ antenna.

Recently, high speed and high data-rate communications have attracted considerable attention. The IEEE $802.15 \mathrm{THz}$ Interest Group (IEEE 802.15 IGthz) has been performing a channel characteristics study for future millimeter and submillimeter indoor wireless communications in the frequency ranges of 75-110 GHz and 270-320 GHz [14].

As one of the important electrical parameters of indoor interior materials, specular reflectance, which is defined as the specular reflection power (i.e., intensity) of a dielectric plate normalized to that of a metal plate, is a prerequisite to the analysis of the channel characteristics for new millimeter and submillimeter indoor wireless communications. Specular reflection, as described by the law of reflection, is the direction of the incident wave and the direction of the reflected wave that make the same angle with respect to the surface normal of an MUT; thus, the angle of incidence is equal to that of reflection $\left(\theta_{i}=\theta_{r}\right)$, as shown in Fig. 1.

This paper describes the specular reflectance measurements of dielectric plates in the $110-325 \mathrm{GHz}$ frequency range. Preliminary results were given in [15], and in this paper, the work is extended by a detailed measurement procedure describing the calibration of a proposed specular reflectance measurement system, comparison of measurement results with those calculated from the analytic solution, and an in-depth analysis of measurement results. Section II briefly describes the analytic solution of the perpendicular-polarized specular reflection of a singlelayer homogeneous isotropic dielectric plate, which is necessary

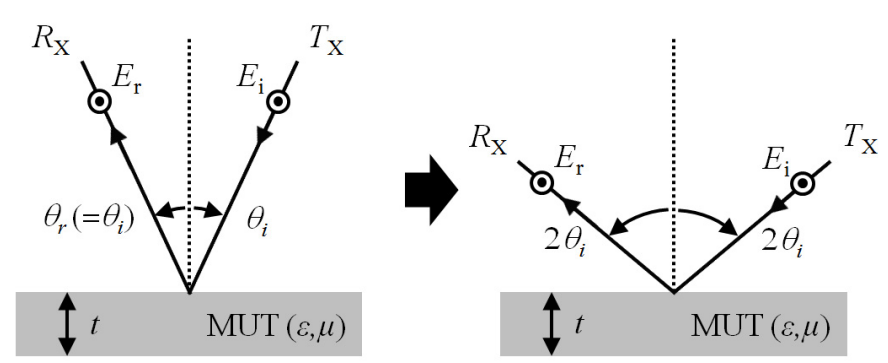

Fig. 1. Conventional specular reflection measurement configuration (MUT is kept fixed, while the Tx part and Rx part, with respect to the surface normal of the MUT, are simultaneously varying with the same speed). to verify measured specular reflectances of MUTs. Sections III and IV describe a proposed specular reflectance measurement system and its measurement procedure, respectively. Section V shows measurement results of the perpendicular-polarized specular reflectance of commonly used dielectric plates and verifies the measured results. Finally, Section VI summarizes this work.

\section{SPECULAR REFLECTION OF A HOMOGENEOUS DiELECTRIC Plate}

When a perpendicular-polarized plane wave is incident upon a single-layer homogeneous isotropic dielectric plate of thickness $t$, as shown in Fig. 1(a), the specular reflection coefficient of the dielectric plate is given by [11]

$$
R_{\text {Specular }}=\frac{E_{\mathrm{r}}\left(\theta_{r}=\theta_{i}\right)}{E_{\mathrm{i}}\left(\theta_{i}\right)}=\frac{1-\exp (-2 j \beta)}{1-R_{\text {Interface }}^{2} \exp (-2 j \beta)} R_{\text {Interface }}
$$

where $\beta=k_{o} t \sqrt{\varepsilon_{r}-\sin ^{2} \theta_{i}}$ is the propagation factor through the dielectric plate, $k_{o}=2 \pi / \lambda_{o}$ is the free-space wavenumber, $\lambda_{o}$ is the free-space wavelength, $\varepsilon_{r}=\varepsilon_{r}^{\prime}-j \varepsilon_{r}^{\prime \prime}$ is the complex relative permittivity of the dielectric plate, $\theta_{i}$ and $\theta_{r}$, respectively, are the incident and reflected angles, and $R_{\text {Interface }}$ is the perpendicular-polarized reflection coefficient at the air-dielectric interface, which is given by

$$
R_{\text {Interface }}=\frac{\cos \theta_{i}-\sqrt{\varepsilon_{r}-\sin ^{2} \theta_{i}}}{\cos \theta_{i}+\sqrt{\varepsilon_{r}-\sin ^{2} \theta_{i}}}
$$

\section{SPECULAR REFLECTANCE MEASUREMENT SYSTEM}

A specular reflectance measurement system is designed to measure both the magnitude and phase of the specular reflection of dielectric plates in the incident angle range of $30^{\circ}-70^{\circ}$ from the surface normal of an MUT in the frequency range for 110-325 GHz. The specular reflectance measurement system consists of an $S$-parameter measuring instrument and a specular reflectance measurement apparatus, as shown in Fig. 2.

The $S$-parameter measuring instrument consists of a $67 \mathrm{GHz}$ vector network analyzer used as the main frame and three frequency extenders which are operating at three waveguide frequency bands, respectively: D-band (110-170 GHz, WR-6.5), G-band (140-220 GHz, WR-5.1), and J-band (220-325 GHz, WR-3.4). The vector network analyzer and frequency extender are connected to each other via phase stable RF and LO cables.

The specular reflectance measurement apparatus, shown in Fig. 2(a), consists of a Tx part, a Rx part, and an MUT holder located at the center of the Tx and Rx parts, where the Tx and $\mathrm{Rx}$ parts consist of a horn antenna and a frequency extender. The Tx and Rx parts and the MUT holder are installed on manual/motorized stages and slides for aligning and fixing them. For adjusting the separation distance between the $\mathrm{Tx}$ and $\mathrm{Rx}$ 


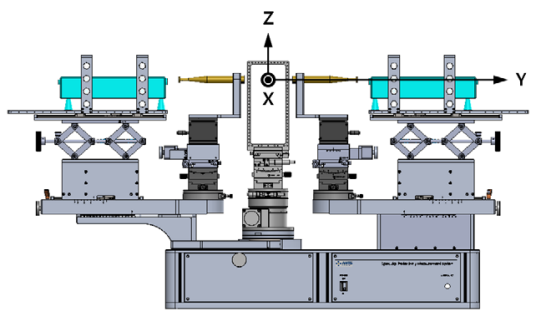

(a)

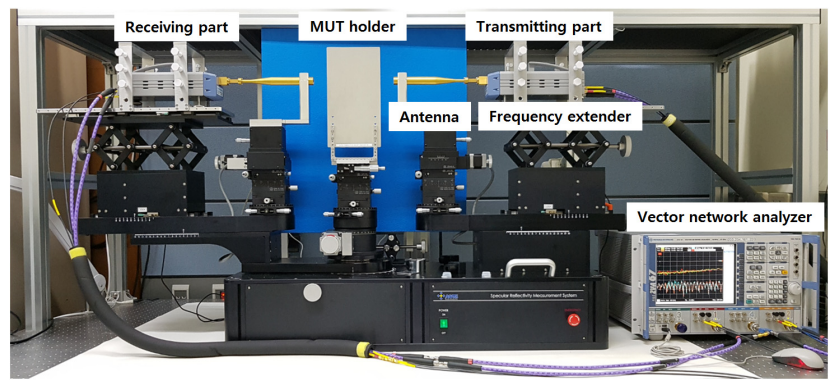

(b)

Fig. 2. (a) Schematic diagram of the specular reflectance measurement apparatus. (b) Implemented specular reflectance measurement system.

antennas and the MUT, a manual Y slide is installed below each Tx and Rx part. Table 1 shows the configuration of the stages and slides and their degrees of freedom, where the $\mathrm{Y}$ and $\mathrm{Z}$ axes in Fig. 2(a) and Table 1 correspond to the line of sight from the MUT toward the Tx antenna and the rotation axis of the MUT holder, respectively.

Usually, during specular reflectance measurements, an MUT is kept fixed, and the angle of the incident wave radiated by the Tx part and that of the reflected wave received by the Rx part, with respect to the surface normal of the MUT, are simultaneously varying with the same speed [16], as depicted in Fig. 1. However, such a specular measurement scheme is not suitable for high frequency ranges, because the $\mathrm{Tx}$ and $\mathrm{Rx}$ parts, consisting of the horn antenna and frequency extender, are bulky. In this case, it is more efficient if one of the Tx and Rx parts is kept fixed, while the other and the MUT is rotated [17]. Here, the Tx part is kept fixed, while the Rx part and the MUT holder are
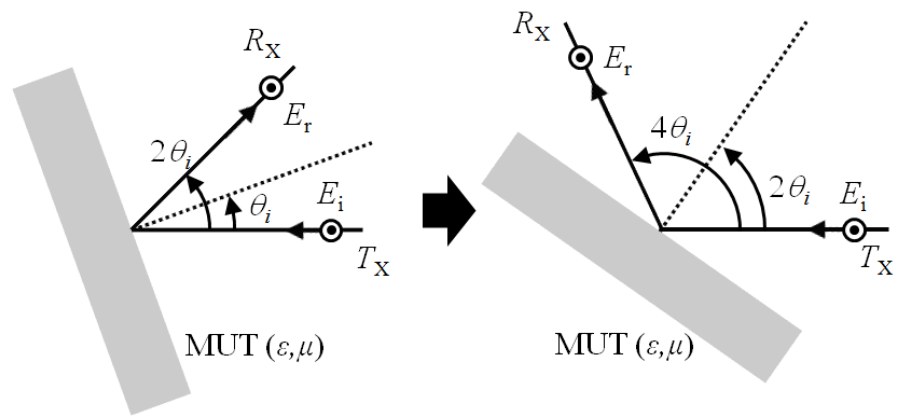

$\operatorname{MUT}(\varepsilon, \mu)$

Fig. 3. Proposed specular reflection measurement configuration (The Tx part is kept fixed, while the Rx part and MUT are concentric-rotating with a $2: 1$ speed ratio).

concentric-rotating with a 2:1 speed ratio for allowing specular reflec-tance measurements, as depicted in Fig. 3, where the rotation angle of the MUT, equal to the angle of the incident wave, is half the rotation angle of the $\mathrm{Rx}$ part.

To minimize the edge diffraction effects of an MUT in freespace specular reflectance measurements without using EM absorbers, a Gaussian-beam-forming corrugated horn antenna, with a nominal power gain of $27 \mathrm{~dB}$ and an axially symmetrical beam with low side lobes, as given in Table 2, is used as Tx and $\mathrm{Rx}$ antennas.

The specular reflectance measurement apparatus is capable of being operated in the incident angle range of $30^{\circ}-70^{\circ}$ from the surface normal of an MUT. The lowest possible angle $\left(30^{\circ}\right)$ is restricted due to the size of the Tx and Rx parts. The highest angle $\left(70^{\circ}\right)$ is ascribed to the MUT size and the Gaussian-beam waste size of the Tx and Rx antennas, as the antenna beam size projected on the surface of an MUT increases with the incident angle.

\section{MEASUREMENT PROCEDURE}

\section{OF SPECULAR REFLECTANCE}

For minimizing the measurement errors inherent in the specular reflectance measurements of an MUT, a measurement procedure may consist of the following steps: 1) alignment of the Tx and Rx antennas and the MUT,2) measurement of the

Table 1. Configuration of stages and slides and their degrees of freedom

\begin{tabular}{|c|c|c|c|c|}
\hline \multirow{2}{*}{ Part name } & \multirow{2}{*}{ Detail } & \multicolumn{2}{|c|}{ Configuration and type of stage and slide } & \multirow{2}{*}{ Degree of freedom } \\
\hline & & Manual & Motorized & \\
\hline \multirow[t]{3}{*}{ Tx part } & Antenna fixture & $\mathrm{Z} / \mathrm{X} / \theta_{\mathrm{x}} / \theta_{\mathrm{y}} /$ rotation stage & Y stage & 6 \\
\hline & Frequency extender fixture & $\theta_{x y} / Z / Y$ slide & - & 3 \\
\hline & Base stage & Y slide & - & 1 \\
\hline MUT holder & MUT fixture & $\mathrm{X} / \mathrm{Y} / \theta_{\mathrm{x}} / \theta_{\mathrm{y}} /$ rotation stage & $Z$ rotation stage & 6 \\
\hline \multirow[t]{3}{*}{$\mathrm{Rx}$ part } & Antenna fixture & $\mathrm{Z} / \mathrm{X} / \theta_{\mathrm{x}} / \theta_{\mathrm{y}} /$ rotation stage & Y stage & 6 \\
\hline & Frequency extender fixture & $\theta_{x y} / Z / Y$ slide & - & 3 \\
\hline & Base stage & Y slide & $Z$ rotation stage & 2 \\
\hline
\end{tabular}

$\mathrm{Y}$ and $\mathrm{Z}$ axes correspond to the line of sight from the MUT toward the Tx antenna and the rotation axis of the MUT holder, respectively. 
Table 2. Specifications of Tx and Rx antennas

\begin{tabular}{|c|c|c|c|c|c|c|}
\hline \multirow{2}{*}{$\begin{array}{l}\text { Type } \\
\text { Features }\end{array}$} & \multicolumn{6}{|c|}{ Gaussian-beam-forming corrugated horn antenna } \\
\hline & \multicolumn{6}{|c|}{ Axially symmetrical beams with low side lobes } \\
\hline Frequency band & \multicolumn{2}{|c|}{ D-band } & \multicolumn{2}{|c|}{ G-band } & \multicolumn{2}{|c|}{ J-band } \\
\hline Operating frequency range $(\mathrm{GHz})$ & \multicolumn{2}{|c|}{$110-170$} & \multicolumn{2}{|c|}{$140-220$} & \multicolumn{2}{|c|}{$220-325$} \\
\hline Aperture diameter $(D, \mathrm{~mm})$ & \multicolumn{2}{|c|}{28} & \multicolumn{2}{|c|}{23} & \multicolumn{2}{|c|}{17} \\
\hline Length (mm) & \multicolumn{2}{|c|}{244} & \multicolumn{2}{|c|}{205} & \multicolumn{2}{|c|}{137} \\
\hline Waveguide connector & \multicolumn{2}{|c|}{ WR-6.5 } & \multicolumn{2}{|c|}{ WR-5.1 } & \multicolumn{2}{|c|}{ WR-3.4 } \\
\hline Gain $(\mathrm{dB})$ & \multicolumn{2}{|c|}{27} & \multicolumn{2}{|c|}{27} & \multicolumn{2}{|c|}{27} \\
\hline Fresnel region $\left(0.62 \sqrt{\frac{D^{3}}{\lambda}}<\mathrm{R}<2 \frac{D^{2}}{\lambda}\right)$ & $\begin{array}{l}110 \mathrm{GHz} \\
140 \mathrm{GHz} \\
170 \mathrm{GHz}\end{array}$ & $\begin{array}{l}56-575 \mathrm{~mm} \\
63-732 \mathrm{~mm} \\
69-889 \mathrm{~mm}\end{array}$ & $\begin{array}{l}140 \mathrm{GHz} \\
170 \mathrm{GHz} \\
220 \mathrm{GHz}\end{array}$ & $\begin{array}{l}47-494 \mathrm{~mm} \\
51-600 \mathrm{~mm} \\
59-776 \mathrm{~mm}\end{array}$ & $\begin{array}{l}220 \mathrm{GHz} \\
260 \mathrm{GHz} \\
325 \mathrm{GHz}\end{array}$ & $\begin{array}{l}37-424 \mathrm{~mm} \\
40-501 \mathrm{~mm} \\
45-626 \mathrm{~mm}\end{array}$ \\
\hline
\end{tabular}

$D$ and $R$ are the largest dimension of an antenna aperture and the separation distance from the antenna, respectively.

specular reflection coefficients of the metal plate used as the reflection standard and the MUT, 3) calculation of the specular reflectance of the MUT, and 4) minimization of multiple reflection effects between the $\mathrm{Tx}$ and $\mathrm{Rx}$ antennas and the MUT on the measured specular reflectances.

\section{Alignment of the Tx and Rx Antennas and the MUT}

1-1) Rotate the Rx part in the opposite direction of the Tx part with respect to the rotation axis of the MUT holder. Set the rotation angle of the $\mathrm{Rx}$ part to $180^{\circ}$ under the assumption that the rotation angle of the Tx part is $0^{\circ}$.

1-2) Install a Gaussian-beam-forming corrugated horn antenna and a frequency extender on both the $\mathrm{Tx}$ and $\mathrm{Rx}$ parts.

1-3) Align the $T_{x}$ and $R x$ antennas to make the two antennas face each other with respect to the rotation axis of the MUT holder.

1-4) Vertically install a rectangular metal plate with a good planarity on the MUT holder, and align the center of the plate surface with the rotation axis of the MUT holder.

1-5) Rotate the metal plate to make the plate surface parallel to the line of sight connecting the two antennas. Set the rotational angle of the MUT holder to $90^{\circ}$.

1-6) Adjust the separation distance between the respective apertures of the $\mathrm{Tx}$ and $\mathrm{Rx}$ antennas and the rotation axis of the MUT holder to be the same.

1-7) Before connecting the antenna to the frequency extender at both the $\mathrm{Tx}$ and $\mathrm{Rx}$ parts, perform a one-port calibration at the test port of the $\mathrm{Tx}$ and $\mathrm{Rx}$ frequency extenders under the measurement conditions of $100 \mathrm{MHz}$ frequency step and $100 \mathrm{~Hz}$ IF bandwidth over an operating frequency range of the frequency extender. Connect the antenna to the test port of the calibrated frequency extender at both Tx and Rx parts.

1-8) Rotate the MUT holder to the rotation angle of $0^{\circ}$ to make the metal plate surface and the Tx antenna face each other.

1-9) Search the rotation angle of the MUT holder where the magnitude of the reflection coefficient $\left(S_{11}^{\mathrm{Tx}}\right)$ of the metal plate at the Tx part is the maximum in the vicinity of $0^{\circ}$.

Set the rotation angle of the MUT holder to $0^{\circ}$.

1-10) Rotate the MUT holder to the rotation angle of $180^{\circ}$ to make the surface of the metal plate and the $\mathrm{Rx}$ antenna face each other.

1-11) Search the rotation angle of the Rx part where the magnitude of the reflection coefficient $\left(S_{22}^{\mathrm{Rx}}\right)$ of the metal plate at the $\mathrm{Rx}$ part is the maximum in the vicinity of $180^{\circ}$. Set the rotation angle of the $\mathrm{Rx}$ part to $180^{\circ}$.

\section{Measurement of the Specular Reflection Coefficients of the} Metal Plate and the MUT

2-1) Measure the forward and backward directional transmission coefficients ( $S_{21}^{\text {metal }}$ and $S_{12}^{\text {metal }}$ ) between the Tx and Rx antennas, which correspond to each directional specular reflection coefficient of the metal plate (theoretically assumed to be -1 ) in the incident angle range of $30^{\circ}-70^{\circ}$ with a $0.5^{\circ}$ step by using the $S$-parameter measuring instrument. The incident angle in the $30^{\circ}-70^{\circ}$ range corresponds to the rotation angle of the MUT holder in the $30^{\circ}$ $-70^{\circ}$ range and that of the $\mathrm{Rx}$ part in the $60^{\circ}-140^{\circ}$ range.

2-2) Rotate the MUT holder and Rx part to the rotation angle of $90^{\circ}$ and $180^{\circ}$, respectively.

2-3) Replace the metal plate with an MUT (a rectangular dielectric plate under test). If the thickness of the MUT is different from that of the metal plate, align the center of the MUT surface with the rotation axis of the MUT holder.

2-4) Measure the forward and backward directional transmission coefficients $\left(S_{21}^{\mathrm{MUT}}\right.$ and $\left.S_{12}^{\mathrm{MUT}}\right)$ between the $\mathrm{Tx}$ and $\mathrm{Rx}$ antennas, which correspond to each directional specular reflection coefficient of the MUT in the incident angle range of $30^{\circ}-70^{\circ}$ with a $0.5^{\circ}$ step by using the $S$-parameter measuring instrument.

\section{Calculation of the Specular Reflectance of the MUT}

3-1) Calculate the forward and backward directional specular reflectance of an MUT, which is defined by the specular re- 
flection power of an MUT normalized to that of the metal plate at each direction, i.e., $\left|S_{21}^{\mathrm{MUT}} / S_{21}^{\text {metal }}\right|^{2}$ and $\left|S_{12}^{\mathrm{MUT}} / S_{12}^{\text {metal }}\right|^{2}$.

3-2) Calculate the specular reflectance $\left(S R_{\text {Specular }}\right)$ of an MUT, which is defined by the average of the forward and backward directional specular reflectance of an MUT:

$$
S R_{\text {Specular }}=\frac{\left.\left|S_{21}^{\mathrm{MUT}} / S_{21}^{\mathrm{metal}}\right|\right|^{2}+\left|S_{12}^{\mathrm{MUT}} / S_{12}^{\text {metal }}\right|^{2}}{2} .
$$

3-3) Repeat four times the specular reflectance measurement, changing the separation distances between the $\mathrm{Tx}$ and $\mathrm{Rx}$ antennas by $\lambda / 8$ intervals (i.e., $d=d_{0}+n \lambda / 8, n=0,1$, 2,3 , where $d, d_{0}$, and $\lambda$ are the separation distance, the initial separation distance, and the wavelength of the signal, respectively).

\section{Minimization of Multiple Reflection Effects between the Tx and Rx Antennas and the MUT on the Measured Specular Reflectances}

4-1) Multiple reflection effects between the Tx and Rx antennas and the MUT can be minimized by averaging out the four measured specular reflectances as follows:

$$
S R_{\text {Specular }}^{\text {Average }}=\frac{1}{4} \sum_{n=0}^{3} S R_{\text {Specular }}\left(d=d_{0}+\frac{n \lambda}{8}\right) .
$$

\section{MEASUREMENT RESULTS}

Perpendicular-polarized specular reflectances of commonly used dielectric plates with a rectangular shape, as shown in Table 3, are measured by using the proposed specular reflectance measurement system in the incident angle of $30^{\circ}-70^{\circ}$ at nine frequencies that are equally selected from the three waveguide frequency bands. The selected nine measurement frequencies are $110 \mathrm{GHz}, 140 \mathrm{GHz}$, and $170 \mathrm{GHz}$ from the D-band; $140 \mathrm{GHz}, 170 \mathrm{GHz}$, and $220 \mathrm{GHz}$ from the G-band; and 220

Table 3. MUT name, its dimension, and the figure number of its measured specular reflectance

\begin{tabular}{lccc}
\hline \multirow{2}{*}{ MUT } & \multicolumn{2}{c}{ Dimension } & \\
\cline { 2 - 3 } & $\begin{array}{c}\text { Thickness } \\
(\mathrm{mm})\end{array}$ & $\begin{array}{c}\text { Width } \times \\
\text { Height } \\
\left(\mathrm{cm}^{2}\right)\end{array}$ & $\begin{array}{c}\text { Figure } \\
\text { number }\end{array}$ \\
\hline Acetal & $2.030,5.644$ & $20 \times 35$ & 5,6 \\
Acryl & $2.010,5.055$ & $20 \times 35$ & 7,8 \\
Bakelite & $2.210,5.163$ & $20 \times 35$ & 9,10 \\
PC (polycarbonate) & $2.010,4.955$ & $20 \times 35$ & 11,12 \\
$\begin{array}{l}\text { PVC (polyvinyl } \\
\text { chloride) }\end{array}$ & $1.730,5.138$ & $20 \times 35$ & 13,14 \\
\hline
\end{tabular}

$\mathrm{GHz}, 260 \mathrm{GHz}$, and $325 \mathrm{GHz}$ from the J-band, where 140 $170 \mathrm{GHz}$ is the overlapped frequency range of the D-/G-bands, and $220 \mathrm{GHz}$ is the boundary frequency of the G-/J-bands.

In the specular reflectance measurements, the separation distance between the respective apertures of the Tx and Rx antennas and the surface of an MUT and a metal plate is $110 \mathrm{~mm}$ in the D-/G-bands and $127 \mathrm{~mm}$ in the J-band, respectively, which means that these reflectance measurements are performed in the Fresnel (or radiating near-field) region of each antenna as shown in Table 2. The Fresnel region is defined by $0.62 \sqrt{\frac{D^{3}}{\lambda}}<$ $R<2 \frac{D^{2}}{\lambda}$, where $D$ and $R$ are the largest dimension of an an tenna aperture and the separation distance from the antenna, respectively [18].

For a rectangular acetal plate of $2.030 \mathrm{~mm}$ thickness and a 20 $\mathrm{cm} \times 35 \mathrm{~cm}$ size, four specular reflectances, which were measured with changing the separation distance between the $\mathrm{Tx}$ and $\mathrm{Rx}$ antennas by $\lambda / 8$ intervals, their averaged-out reflectance, and the analytic reflectance are shown at $140 \mathrm{GHz}$ of the G-band and $220 \mathrm{GHz}$ of the J-band as depicted in Fig. 4(a) and (b), respectively. Fig. 4 shows at the two frequencies that (1) multiple reflection effects between the Tx and Rx antennas and the MUT on the measured specular reflectances can be minimized by averaging out the multiple specular reflectances measured at the different separation distances by $\lambda / 8$ intervals, and (2) the measured specular reflectances can be verified by the fact that the averaged-out results (green line with dot) and the theoretical ones (black dot) agree well with each other. This means that the specular reflectance measurement system can be effectively calibrated to minimize the measurement errors inherent in the measurement system in the Fresnel region of Tx and Rx antennas. The theoretical specular reflectances are calculated by using (1) and (2) above with the complex relative permittivity of acetal with $2.030 \mathrm{~mm}$ thickness, in this case $\varepsilon_{r}=2.800-j 0.043$, which is measured by using a quasi-optic free-space material measurement system $[8,9]$.

Measured specular reflectances of commonly used dielectric plates, as listed in Table 3, are shown in Figs. 5-14, whose upper,

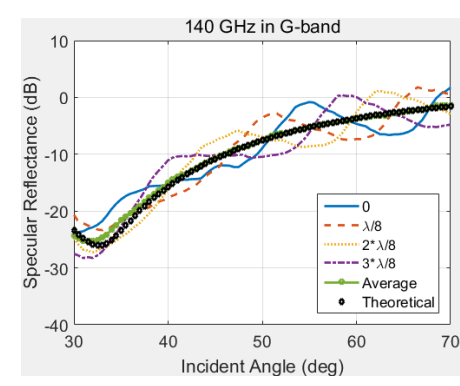

(a)

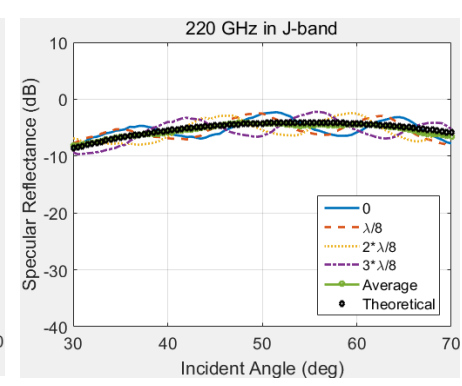

(b)
Fig. 4. Specular reflectance of an acetal plate of $2.030 \mathrm{~mm}$ thickness. (a) $140 \mathrm{GHz}$ of the G-band. (b) $220 \mathrm{GHz}$ of the J-band. 

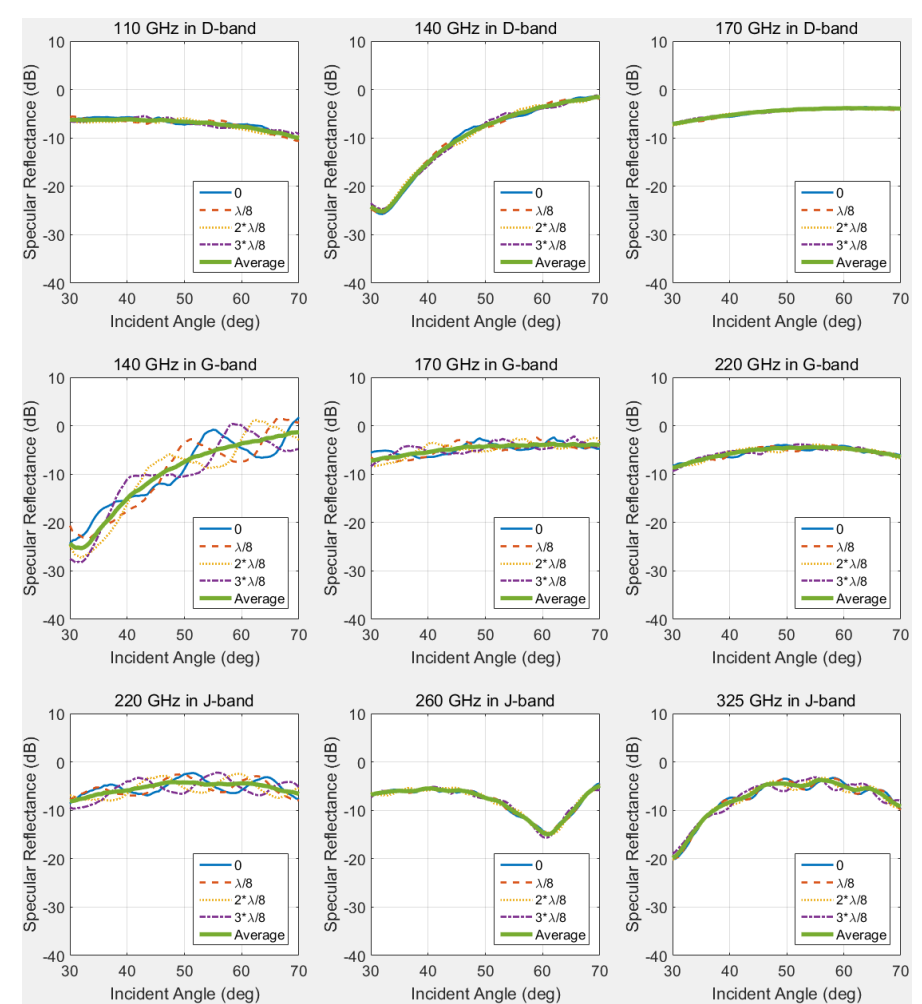

Fig. 5. Specular reflectance of an acetal plate of $2.030 \mathrm{~mm}$ thickness.

middle, and lower correspond to the specular reflectances measured in the D-/G-/J-bands, respectively. Regardless of the kind of MUTs, Figs. 5-14 show, in the Fresnel region of Tx and Rx antennas, that 1) multiple reflection effects between the two
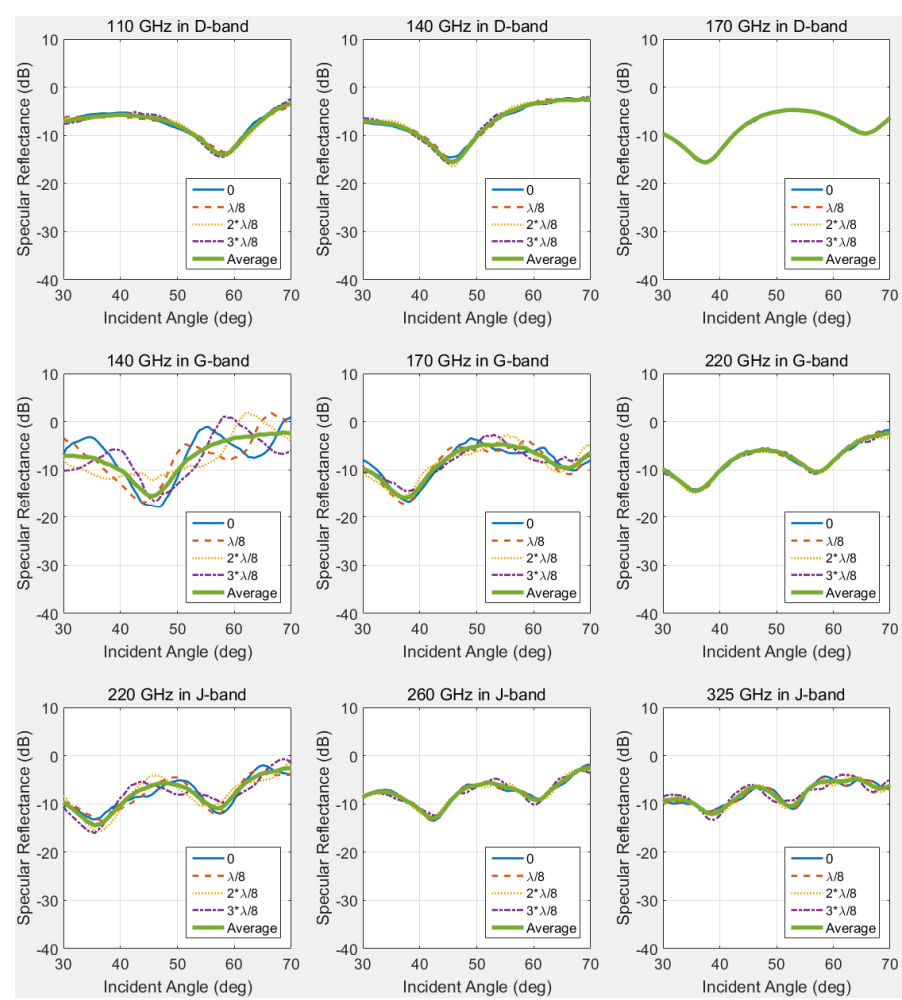

Fig. 6. Specular reflectance of an acetal plate of $5.644 \mathrm{~mm}$ thickness.
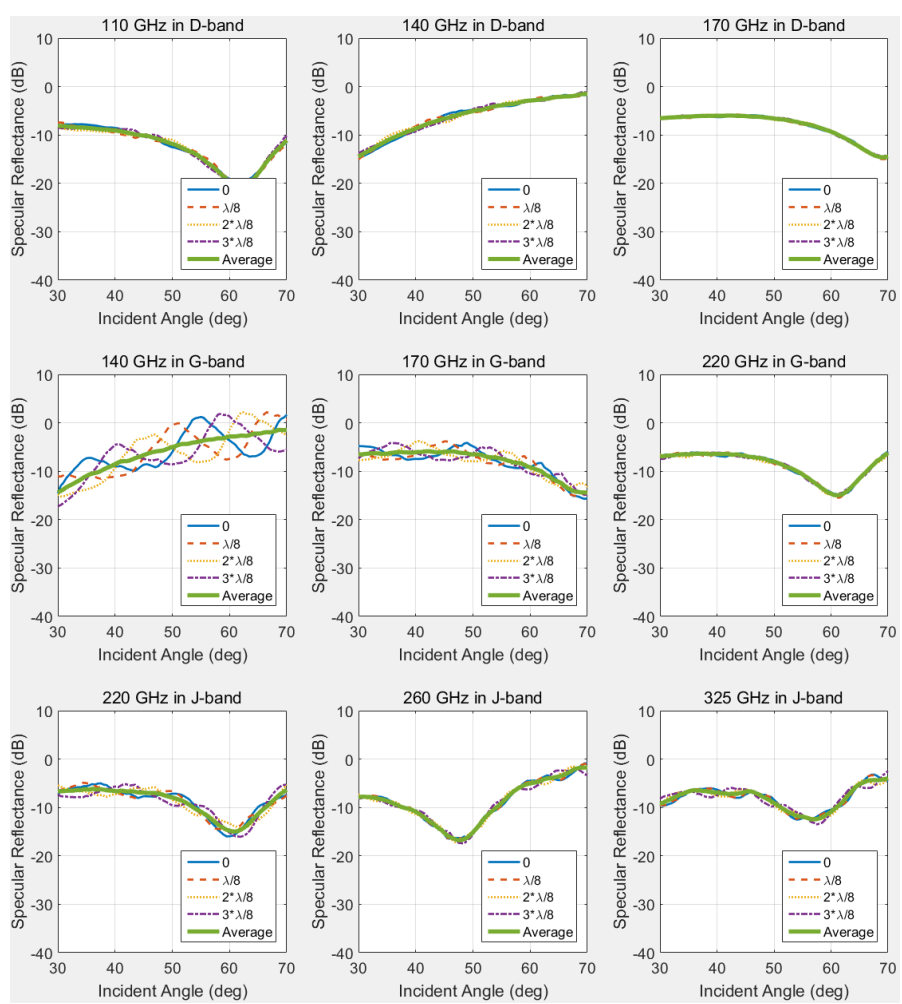

Fig. 7. Specular reflectance of an acryl plate of $2.010 \mathrm{~mm}$ thickness.

antennas and the MUT can be minimized by averaging out the multiple specular reflectances measured at the different separation distances by $\lambda / 8$ intervals, 2) the multiple reflection effects in the D-band are weaker than in the G-/J-bands, and 3) the
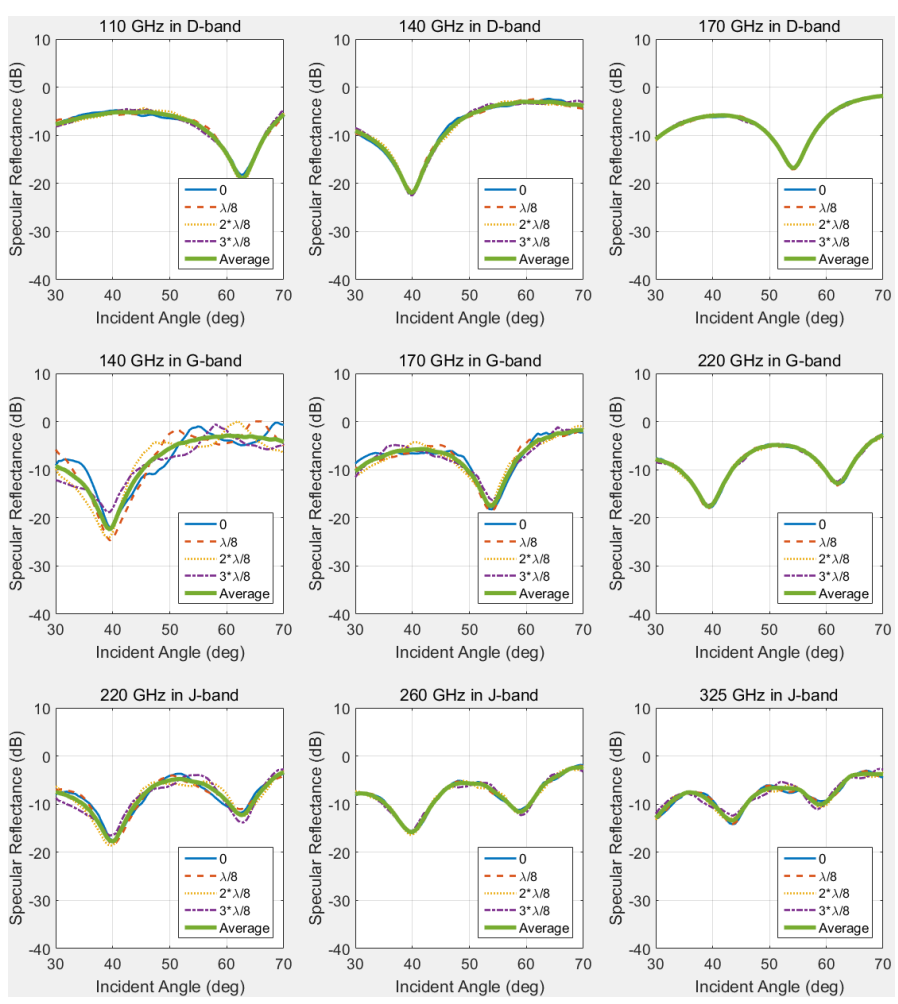

Fig. 8. Specular reflectance of an acryl plate of $5.055 \mathrm{~mm}$ thickness. 

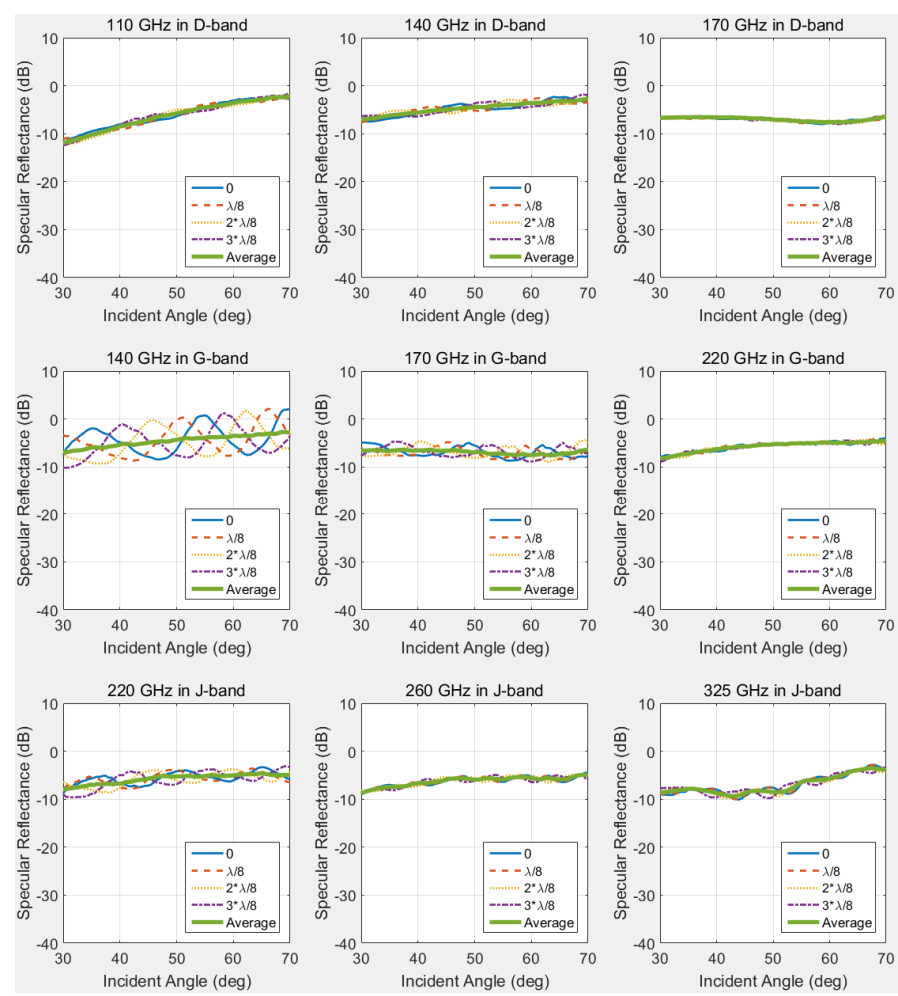

Fig. 9. Specular reflectance of a bakelite plate of $2.210 \mathrm{~mm}$ thickness.

measured specular reflectances can be verified by the fact that the measured results over the two overlapped frequencies of the D-/G-band, i.e., $140 \mathrm{GHz}$ (the center of the upper and the left of the middle in Figs. 5-14) and $170 \mathrm{GHz}$ (the right of the up-
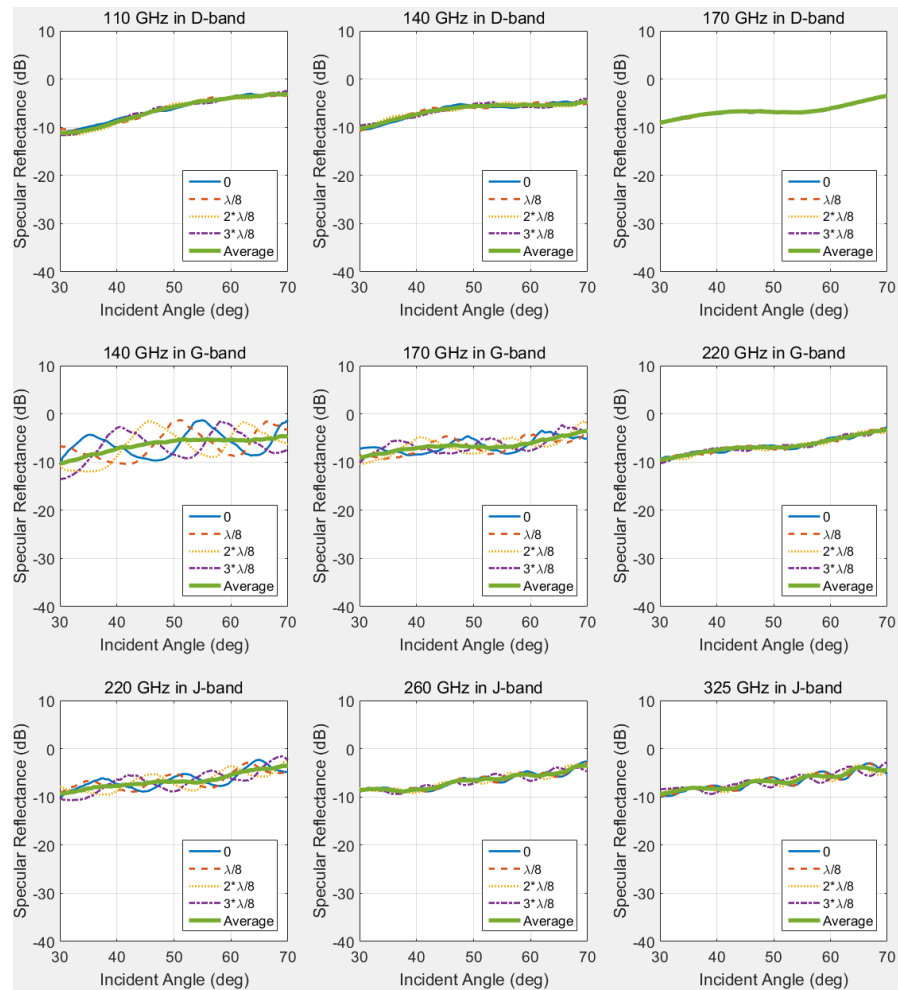

Fig. 10. Specular reflectance of a bakelite plate of $5.163 \mathrm{~mm}$ thickness.
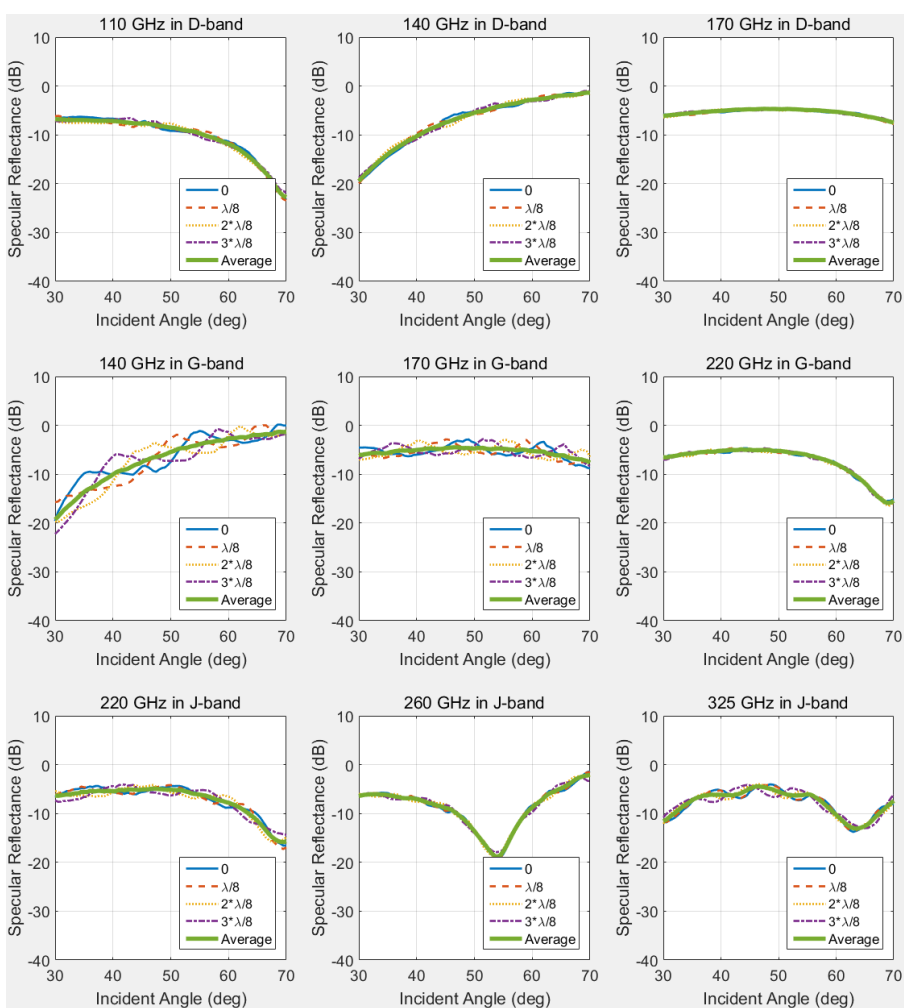

Fig. 11. Specular reflectance of a PC plate of $2.010 \mathrm{~mm}$ thickness.

per and the center of the middle), and at the boundary frequency of the G-/J-band, i.e., $220 \mathrm{GHz}$ (the right of the middle and the left of the lower), agree well with the results for the other band, respectively.
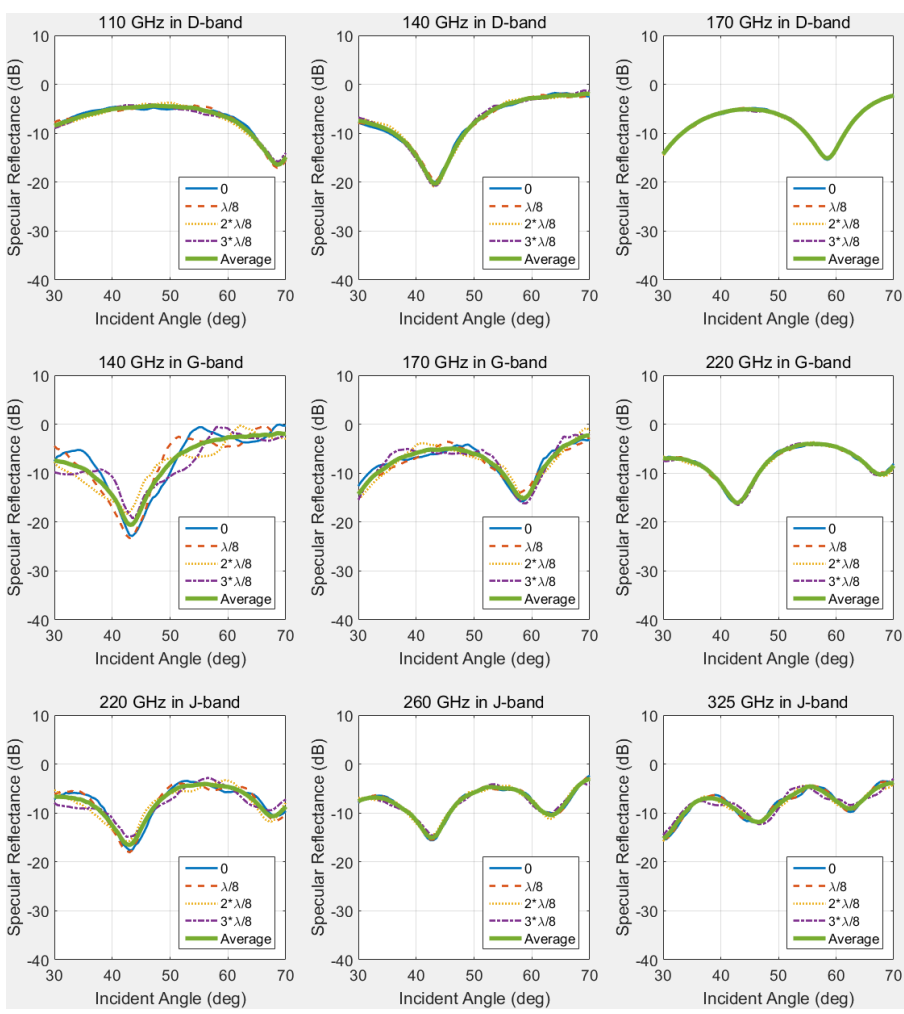

Fig. 12. Specular reflectance of a PC plate of $4.955 \mathrm{~mm}$ thickness. 

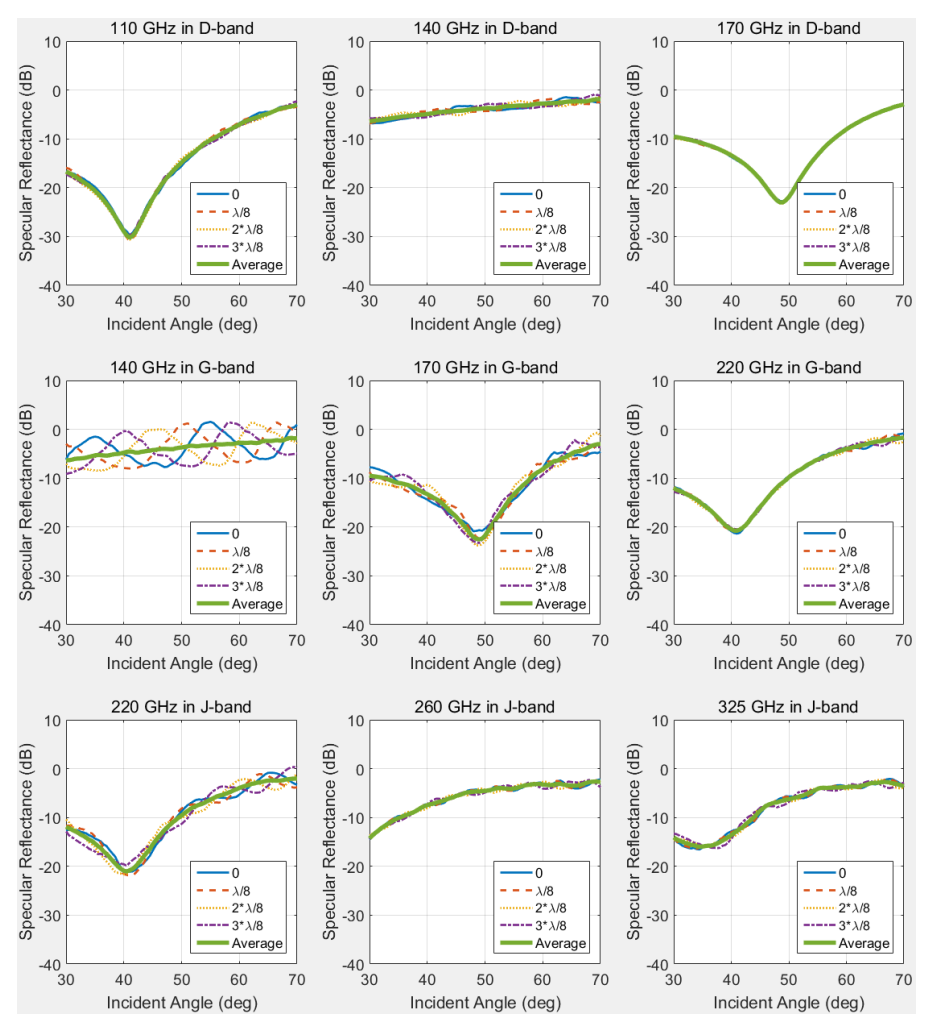

Fig. 13. Specular reflectance of a PVC plate of $1.730 \mathrm{~mm}$ thickness.
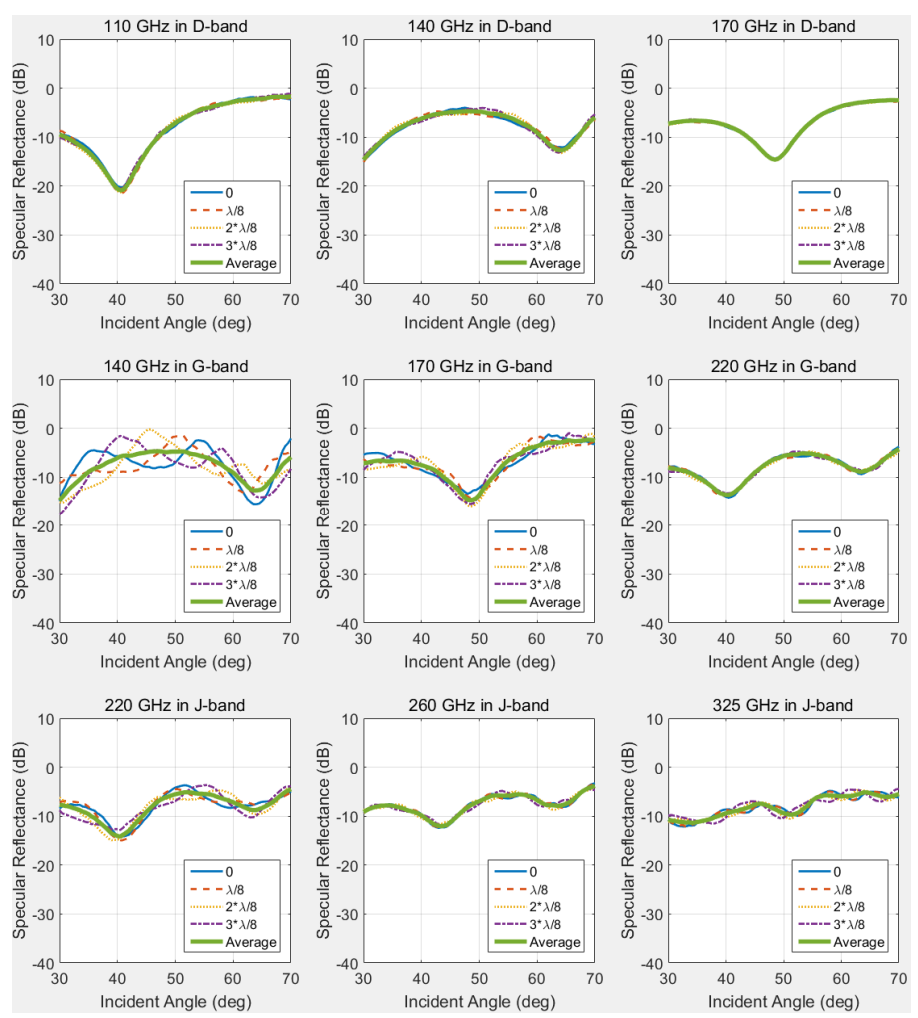

Fig. 14. Specular reflectance of a PVC plate of $5.138 \mathrm{~mm}$ thickness.

\section{CONCLUDING REMARKS}

In this paper, specular reflectance measurements of dielectric plates are described in the $110-325 \mathrm{GHz}$ frequency range, i.e., three waveguide frequency bands: D-band (110-170 GHz), Gband (140-220 GHz), and J-band (220-325 GHz).

The proposed specular reflectance measurement system consists of an $S$-parameter measuring instrument and a specular reflectance measurement apparatus. The Tx part of the specular reflectance measurement apparatus is kept fixed, while the $\mathrm{Rx}$ part and the MUT holder are concentric-rotating with a 2:1 speed ratio for allowing specular reflectance measurements.

The specular reflectance measurement system is effectively calibrated to minimize the measurement errors inherent in the measurement system in the following ways: 1) by using a Gaussian-beam-forming corrugated horn antenna with an axially symmetrical beam with low side lobes to minimize the edge diffraction effects of an MUT in free-space measurements without using EM absorbers, 2) by normalizing the specular reflection power of an MUT to that of a metal plate used as the reflection standard, and 3) by averaging out the multiple specular reflectances measured with changing the separation distance between the $\mathrm{Tx}$ and $\mathrm{Rx}$ antennas by $\lambda / 8$ intervals to minimize the multiple reflection effects between the two antennas and an MUT on the measured specular reflectances.

Measurement results of the perpendicular-polarized specular reflectance of commonly used dielectric plates are verified in the Fresnel region of $\mathrm{Tx}$ and $\mathrm{Rx}$ antennas by the fact that 1), for acetal, the average of the multiple specular reflectances measured with changing the separation distance between the $\mathrm{Tx}$ and $\mathrm{Rx}$ antennas by $\lambda / 8$ intervals agrees well with the analytic result, and 2) the results measured at the two overlapped frequencies of the D-/G-bands, i.e., $140 \mathrm{GHz}$ and $170 \mathrm{GHz}$, and at the boundary frequency of the G-/J-bands, i.e., $220 \mathrm{GHz}$, agree well with the results at the other band for all of MUTs, respectively.

\section{REFERENCES}

[1] T. M. Nguyen and J. Y. Chung, "RF conductivity measurement of conductive Zell fabric," Journal of Electromagnetic Engineering and Science, vol. 16, no. 1, pp. 24-28, 2016.

[2] G. Y. Kim and B. S. Lee, "Synthesis of bulk medium with negative permeability using ring resonators," Journal of Electromagnetic Engineering and Science, vol. 16, no. 2, pp. 6773, 2016.

[3] B. Komiyama, M. Kiyokawa, and T. Matsui, "Open resonator for precision dielectric measurements in the $100 \mathrm{GHz}$ band," IEEE Transactions on Microwave Theory and Techniques, vol. 39, no. 10, pp. 1792-1796, 1991.

[4] S. N. Dudorov, D. V. Lioubtchenko, J. A. Mallat, and A. V. Raisanen, "Differential open resonator method for permittivity measurements of thin dielectric film on substrate," IEEE Transactions on Instrumentation and Measurement, vol. 54, no. 5, pp. 1916-1920, 2005. 
[5] D. K. Ghodgaonkar, V. V. Varadan, and V. K. Varadan, "Freespace measurement of complex permittivity and complex permeability of magnetic materials at microwave frequencies," IEEE Transactions on Instrumentation and Measurement, vol. 39, no. 2, pp. 387-394, 1990.

[6] D. Bourreau, A. Peden, and S. Le Maguer, "A quasi-optical free-space measurement setup without time-domain gating for material characterization in the W-band," IEEE Transactions on Instrumentation and Measurement, vol. 55, no. 6, pp. 2022-2028, 2006.

[7] A. Kazemipour, M. Hudlicka, S. K. Yee, M. A. Salhi, D. Allal, T. Kleine-Ostmann, and T. Schrader, "Design and calibration of a compact quasi-optical system for material characterization in millimeter/submillimeter wave domain," IEEE Transactions on Instrumentation and Measurement, vol. 64, no. 6, pp. 1438-1445, 2015.

[8] J. S. Kang, J. H. Kim, C. H. Cho, and D. C. Kim, "Wband permittivity measurements using a free-space material measurement technique," The Journal of Korean Institute of Electromagnetic Engineering and Science, vol. 24, no. 3, pp. 253-258, 2013.

[9] J. S. Kang, J. H. Kim, and J. I. Park, "Reduction of edge diffraction effects of MUT holder using EM absorber in W-band free-space material measurements," in Proceedings of the 2016 International Symposium on Antennas and Propagation (ISAP), Okinawa, Japan, 2016, pp. 936-937.

[10] S. Iyer, C. C. Cheng, C. Kim, and A. Abbaspour-Tamijani, "Compact Gaussian beam system for S-parameter characterization of planar structures at millimeter-wave frequencies," IEEE Transactions on Antennas and Propagation, vol. 59, no. 9, pp. 2437-2444, 2010.

[11] F. Sagnard, F. Bentabet, and C. Vignat, "In situ measurements of the complex permittivity of materials using reflection ellipsometry in the microwave band: theory (Part I),"

Jin-Seob Kang

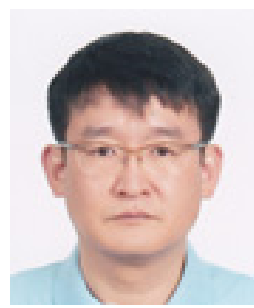

received the B.S. degree in electronic engineering from Hanyang University, Seoul, Korea, in 1987, and the M.S. and Ph.D. degrees in electrical engineering from Korea Advanced Institute of Science and Technology (KAIST), Daejeon, Korea, in 1989 and 1994, respectively. He was a Postdoctoral Research Associate with the Department of Electrical and Computer Engineering, University of Illinois at Urbana-Champaign, in 1995 and an Assistant Professor with the School of Electrical and Electronic Engineering, Chungbuk National University, Korea, from 1996 to 1997. He joined the Korea Research Institute of Standards and Science, Daejeon, in 1998. His research interests include electromagnetics measurement standards (scattering parameters, antenna characteristics, field strength, material parameters) and (sub-)mm-wave measurements.
IEEE Transactions on Instrumentation and Measurement, vol. 54, no. 3, pp. 1266-1273, 2005.

[12] F. Sagnard, F. Bentabet, and C. Vignat, "In situ measurements of the complex permittivity of materials using reflection ellipsometry in the microwave band: theory (Part II)," IEEE Transactions on Instrumentation and Measurement, vol. 54, no. 3, pp. 1274-1282, 2005.

[13] M. Klenner, C. Zech, A. Hulsmann, J. Kuhn, M. Schlechtweg, and O. Ambacher, "Spectroscopic measurement of material properties using an improved millimeterwave ellipsometer based on metallic substrates," IEEE Transactions on Instrumentation and Measurement, vol. 65, no. 11, pp. 2551-2559, 2016.

[14] IEEE 802.15 WPAN Terahertz Interest Group (IGthz) [Online]. Available: http://www.ieee802.org/15/pub/IGthzOLD.html.

[15] J. S. Kang, J. H. Kim, K. Y. Kang, D. H. Yoon, and S. W. Park, "Specular reflectance measurement of dielectric plates in 110-325 GHz frequency range," in Proceedings of the 39th Annual Meeting and Symposium of the Antenna Measurement Techniques Association (AMTA), Atlanta, GA, 2017.

[16] M. H. Umari, D. K. Ghodgaonkar, V. V. Varadan, and V. K. Varadan, "A free-space bistatic calibration technique for the measurement of parallel and perpendicular reflection coefficients of planar samples," IEEE Transactions on Instrumentation and Measurement, vol. 40, no. 1, pp. 19-24, 1991.

[17] X. Faget, N. Mallejac, and A. Litman, "Bistatic free-space measurements of magneto-dielectric materials and comparison with numerical models," in Proceedings of the 2016 URSI International Symposium on Electromagnetic Theory (EMTS), Espoo, Finland, 2016, pp. 404-406.

[18] C. A. Balanis, Modern Antenna Handbook. New York, NY: John Wiley \& Sons, 2008.

Jeong-Hwan Kim

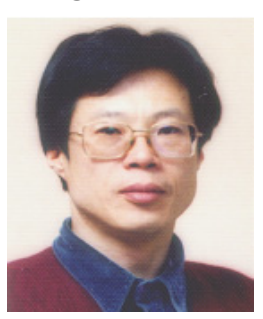

was born in Cheongju, Korea in 1954. He received the B.S. degree in electrical engineering from Seoul National University, Seoul, Korea in 1978, and the M.S. and the Ph.D. degrees from Korea Advanced Institute of Science and Technology, Seoul, Korea in 1980 and 2000, respectively, both in electrical and electronic engineering. He joined the Center for Electromagnetic Wave, the Korea Research Institute of Standards and Science, Daedeok Science Town, Korea in 1981. Since then, he has been working on the developments of the six-port ANA systems, standard antennas, and electromagnetics measurement standards. 


\section{Kwang-Yong Kang}

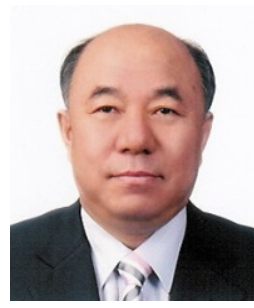

was born in Sacheon, Korea in 1951. He received the B.S. degree from Seoul National University, Seoul, Korea, in 1975, and the Ph.D. degree in solid-state physics from Pusan National University, Busan, Korea, in 1988. Since 1989, he has been a member of engineering staff of the ETRI, Daejeon, Korea. He was a member of the IEEE MTT and OSA of USA, JJAP of Japan, and KEES/KPS of Korea. He retired from the institute (ETRI), passed through the university, and he is working for a research institute of a small company, Panoptics Corp. He is now continuing his research work ( $\mathrm{THz}$ field) at the Lab. of the Corporate Research Lab. His research interests include the HTS thin films and millimeter-wave device, metal-insulator transition (MIT) devices, and terahertz technologies.

\section{Dae-Hwan Yoon}

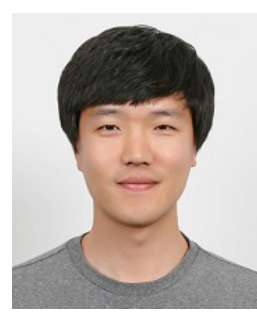

received the B.S. degree in electronic engineering from Chosun University, Gwangju, Korea, in 2007. He joined the Gwangju Radio Management Service, Naju, Korea, from 2008 to 2014. He joined the National Radio Research Agency, Naju, Korea, in 2015.

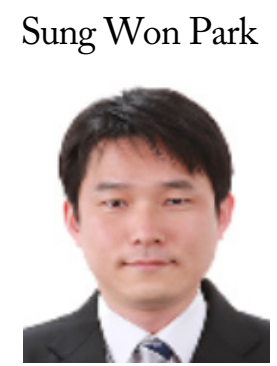

received the M.S. degree in information and communications engineering from Kyunghee University, Seoul, Korea, in 2003. He joined the National Radio Research Agency, Naju, Korea, in 2003 and in 2013, he was promoted to Deputy Director. 